\title{
Virasoro vacuum block at next-to-leading order in the heavy-light limit
}

\author{
Matteo Beccaria, Alberto Fachechi and Guido Macorini \\ Dipartimento di Matematica e Fisica Ennio De Giorgi, \\ Università del Salento \& INFN, Via Arnesano, 73100 Lecce, Italy \\ E-mail: matteo.beccaria@le.infn.it, alberto.fachechi@le.infn.it, \\ macorini@nbi.ku.dk
}

ABSTRACT: We consider the semiclassical limit of the vacuum Virasoro block describing the diagonal 4-point correlation functions on the sphere. At large central charge $c$, after exponentiation, it depends on two fixed ratios $h_{\mathrm{H}} / c$ and $h_{\mathrm{L}} / c$, where $h_{\mathrm{H}, \mathrm{L}}$ are the conformal dimensions of the 4-point function operators. The semiclassical block may be expanded in powers of the light ratio $h_{\mathrm{L}} / c$ and the leading non-trivial (linear) order is known in closed form as a function of $h_{\mathrm{H}} / c$. Recently, this contribution has been matched against $\mathrm{AdS}_{3}$ gravity calculations where heavy operators build up a classical geometry corresponding to a BTZ black hole, while the light operators are described by a geodesic in this background. Here, we compute for the first time the next-to-leading quadratic correction $\mathcal{O}\left(\left(h_{\mathrm{L}} / c\right)^{2}\right)$, again in closed form for generic heavy operator ratio $h_{\mathrm{H}} / c$. The result is a highly nontrivial extension of the leading order and may be relevant for further refined $\mathrm{AdS}_{3} / \mathrm{CFT}_{2}$ tests. Applications to the two-interval Rényi entropy are also presented.

Keywords: AdS-CFT Correspondence, Conformal and W Symmetry, Field Theories in Lower Dimensions

ARXIV EPRINT: 1511.05452 


\section{Contents}

1 Introduction 1

2 Notation and preliminary considerations 3

3 Semiclassical limit $\quad 5$

3.1 Exact expansions from the recursion relations 6

4 The exact expression for the NLO contribution $f^{(2)}(\lambda, z) \quad 8$

4.1 Exact expression for $f^{(2,2)}(z)$ and $f^{(2,3)}(z) \quad 8$

5 A simple application: two interval Rényi entropy 9

6 Conclusions 11

A Long expansion for the function $f^{(2)}(\alpha, z) \quad 12$

B Details of the derivation $\quad 13$

$\begin{array}{ll}\text { B.1 The monodromy problem } & 13\end{array}$

$\begin{array}{ll}\text { B.2 Special cases } & 14\end{array}$

B.3 Back to the monodromy problem 16

C Expressions of $c_{2}(\alpha, z)$ at $\alpha=\frac{1}{4}, \frac{1}{6} \quad 18$

$\begin{array}{ll}\text { D Long expansion of the two-interval Rényi entropy } & 18\end{array}$

\section{Introduction}

Conformal field theories are characterised by their operator spectrum and fusion rules. From this basic data, correlation functions may be computed by repeatedly applying the operator product expansion (OPE). This procedure is conveniently organised with respect to the representation content of the conformal algebra. Its main ingredients are the global conformal blocks that are fully determined by conformal symmetry [1-7]. In two dimensions, the global conformal algebra is enhanced to the Virasoro algebra Vir $\oplus \overline{\text { Vir }}$ and the associated decomposition of correlation functions in terms of Virasoro conformal blocks is a central problem in the study of dynamical properties. In particular, knowledge of Virasoro conformal blocks is important for the bootstrap program [8], in Liouville theory [9], AGT correspondence $[10,11]$, and, of course, in the context of $\mathrm{AdS}_{3} / \mathrm{CFT}_{2}$ duality as we shall discuss later.

In this paper, we focus on the determination of the vacuum Virasoro conformal blocks for the 4-point correlation functions on the sphere. Virasoro blocks encode the contributions from the virtual exchange of the states associated with a given (internal) conformal family. 
They are functions $\mathcal{F}\left(c, \boldsymbol{h}, h_{p}, z\right)$ that depend on the central charge $c$, the external and internal operators conformal weights $\boldsymbol{h}=\left\{h_{i}\right\}_{i=1, \ldots, 4}$ and $h_{p}$, and the conformally invariant cross-ratio $z$ of the four points.

Virasoro blocks are known in closed form only in a few special cases, typically involving degenerate fields [12]. Series expansions in various scattering channels may be derived by brute force computation exploiting the Virasoro algebra or by the recursion relations derived by Zamolodchikov [13, 14]. Recently, a remarkable progress in this direction has been achieved in [15] where three different closed-form perturbative expansions have been obtained for the 4-point Virasoro blocks on the sphere. These representations are an important computational tool to examine the semiclassical regime, i.e. a large central charge limit where $c \rightarrow \infty$ with some of the ratios $h_{i} / c$ being fixed [16]. Of course, one of the main motivations for considering this limit is that it is suitable for a holographic study by means of the AdS/CFT duality. Following standard usage, the operators with growing conformal dimension $h \sim c$ are called heavy, while operators with fixed $h$ are called light. An important and well studied tractable case is the heavy-light limit [17] associated with the following 4-point function

$$
\text { Heavy-Light: } \quad\left\langle\Phi_{H} \Phi_{H} \Phi_{L} \Phi_{L}\right\rangle .
$$

In this case, the relevant Virasoro block is the one associated with the conformal family of the identity operator, i.e. the so-called vacuum block. The $\mathrm{AdS}_{3}$ bulk interpretation is that the heavy operators build up a classical asymptotically $\mathrm{AdS}_{3}$ geometry corresponding to a BTZ black hole [18], while the light operators are described by a geodesic probing this background solution. The heavy-light case (1.1) may be generalised in various ways $[17,19-$ 22]. In particular, it is interesting to regard it as a special case of the general fully-heavy situation where all ratios $h_{i} / c$ are fixed as $c \rightarrow \infty$, but two of them are taken to be small in the end. This is legitimate because it has been proved that there is no problem with the order of limits [20]. Recently, this holographic computation of the semiclassical 4-point function at first order in $h_{\mathrm{L}} / c$ has been fully clarified in [23]. To this aim, a bulk construction has been presented based on the geodesic Witten diagrams recently introduced in [24], evaluated in locally $\mathrm{AdS}_{3}$ geometries generated by the back-reaction of the heavy operators.

From the point of view of the $2 \mathrm{~d}$ boundary CFT, the semiclassical limit is well defined due to the exponentiation property of the Virasoro blocks [5, 9, 13, 25]. Indeed, at large central charge we have

$$
\log \mathcal{F}\left(c, \boldsymbol{h}, h_{p}, z\right)=-\frac{c}{6} f\left(\boldsymbol{\lambda}, \lambda_{p}, z\right)+\mathcal{O}\left(c^{0}\right),
$$

where $\lambda_{i}$ are the fixed ratios $h_{i} / c$. The function $f$ is sometimes referred as the classical conformal block. In the bulk $\mathrm{AdS}_{3}$ gravity theory, this classical part describes relativistic massive particles probing the asymptotically $\mathrm{AdS}_{3}$ geometry. While a closed form is not known, we can study this function perturbatively with respect to the small (fixed) ratios $h_{\mathrm{L}} / c$, each term being determined by a monodromy prescription. More precisely, the first term of this expansion describes light particles propagating along the geodesics of the geometry generated by the heavy operators. The next-to-leading order accounts for 
the back-reaction of particles on the geometry and describes the corrections to the emergent thermality of heavy microstates at large $c[20]$. Further subleading terms in the $1 / c$ expansion (1.2), which are invisible in the semiclassical limit, account for truly quantum gravitational corrections which have been recently investigated in [26]. In $\mathrm{AdS}_{3}$ bulk theory, one can compute these corrections holographically by including the contributions of gravitons 1-loop to geodesic Witten diagrams.

We now specialise to the subject of this paper, i.e. the vacuum Virasoro conformal block that contains the contributions from the identity conformal family with $h_{p}=0$. In this case, fusion onto the identity requires to consider a pairwise correlator with $h_{1}=h_{2}$ and $h_{3}=h_{4}$. The function in the r.h.s. of (1.2) is then $f\left(\lambda_{1}, \lambda_{3}, z\right)$, symmetric under the exchange $\lambda_{1} \leftrightarrow \lambda_{3}$. According to the above discussion, the interest in AdS/CFT tests suggests to consider the following expansion around $\lambda_{1}=0$, with generic $\lambda_{3}$

$$
f\left(\lambda_{1}, \lambda_{3}, z\right)=\lambda_{1} f^{(1)}\left(\lambda_{3}, z\right)+\lambda_{1}^{2} f^{(2)}\left(\lambda_{3}, z\right)+\cdots .
$$

The first correction $f^{(1)}\left(\lambda_{3}, z\right)$ has been computed in [17] in closed form. The calculation is based on the perturbative solution of a monodromy problem $[9,27]$ (see also the more recent works [28-30]). ${ }^{1}$ The holographic setup in [23] is indeed able to reproduce $f^{(1)}\left(\lambda_{3}, z\right)$ by means of a bulk gravity calculation. In order to sharpen both this picture and the associated tools, we believe that it is important to extend the expansion (1.3) including the much more difficult next order contribution $f^{(2)}\left(\lambda_{3}, z\right)$. In principle, this task can be accomplished by solving the monodromy problem at higher order, but technical difficulties must be overcome. The aim of this paper is precisely that of providing the exact expression for $f^{(2)}\left(\lambda_{3}, z\right)$. Although our derivation will be somewhat heuristic, the final result presented in (4.1) - passes several non-trivial tests. In particular, it agrees with the explicit calculation of the perturbative Virasoro vacuum block at large orders in the $s$ channel, that we have computed exploiting the powerful recent results of [15].

The plan of the paper is the following. In section 2 we set up the notation. In section 3 we discuss the semiclassical limit of the vacuum block in some details. We include explicit results from the high order solution of the recursion relations obeyed by the block. This allows to derive a series expansion of the (perturbative) exponentiated block. In section 4 we present our main results. Finally, in section 5 we give a simple application to the study of two-interval mutual Rényi information. The details of our derivation and some additional exact results are collected in appendix B. Other appendices collect long expressions that may be useful for the reader.

\section{Notation and preliminary considerations}

The general form of the OPE between two Virasoro primary fields $\Phi_{1}, \Phi_{2}$ with conformal dimensions $h_{1}, h_{2}$ is

$$
\Phi_{1}(z, \bar{z}) \Phi_{2}(0,0)=\sum_{p} C_{12}^{p}|z|^{2\left(h_{p}-h_{1}-h_{2}\right)} \Psi_{p}(z, \bar{z} \mid 0,0),
$$

\footnotetext{
${ }^{1}$ In the context of holographic calculations of entanglement entropy [27, 31], the monodromy approach is related to the problem of finding solutions of Einstein equations with given boundary behaviour.
} 
where we sum over all primaries $\Phi_{p}(z, \bar{z})$ and where $\Psi_{p}(z, \bar{z} \mid 0,0)$ takes into account the contributions from the conformal family of $\Phi_{p}(z, \bar{z})$, i.e. the primary and its descendants. The explicit expression of $\Psi_{p}(z, \bar{z} \mid 0,0)$ involves a sum over descendants and may be completely fixed by using the Virasoro algebra (see [32] for efficient algorithms). The 3-point couplings $C_{12}^{p}$ are among the basic CFT data. A 4-point correlation function of primaries has indeed the form

$$
\left\langle\Phi_{1}(\infty) \Phi_{2}(1) \Phi_{3}(z) \Phi_{4}(0)\right\rangle=\frac{1}{|z|^{2\left(h_{3}+h_{4}\right)}} \sum_{p} C_{12, p} C_{34}^{p}\left|\mathcal{F}\left(c, \boldsymbol{h}, h_{p}, z\right)\right|^{2},
$$

where we used global conformal invariance to fix three operators at $\infty, 1,0$. The letter $\boldsymbol{h}$ stands collectively for the conformal dimensions $h_{1}, \ldots, h_{4}$, and the index in the fusion coefficients is raised by the Zamolodchikov metric $g_{a b}=\left\langle\Phi_{a} \mid \Phi_{b}\right\rangle$. The Virasoro block associated with the internal primary $\Phi_{p}(z, \bar{z})$ is the function $\mathcal{F}\left(c, \boldsymbol{h}, h_{p}, z\right)$ in (2.2). It may be expanded in powers of $z$, i.e. in the Virasoro level $\ell$

$$
\mathcal{F}\left(c, \boldsymbol{h}, h_{p}, z\right)=z^{h_{p}} \sum_{\ell=0}^{\infty} \mathcal{F}_{\ell}\left(c, \boldsymbol{h}, h_{p}\right) z^{\ell} .
$$

This expression may be further refined by separating the contributions of the level $q$ quasiprimaries with respect to the global conformal algebra $\mathfrak{s l}(2, \mathbb{R}) \oplus \mathfrak{s l}(2, \mathbb{R})$, see [15] for a clean presentation. The result is $\left(h_{i j} \equiv h_{i}-h_{j}\right)$

$$
\mathcal{F}\left(c, \boldsymbol{h}, h_{p}, z\right)=z^{h_{p}} \sum_{q=0}^{\infty} \chi_{q}\left(c, \boldsymbol{h}, h_{p}\right) z^{q}{ }_{2} F_{1}\left(h_{p}+q+h_{12}, h_{p}+q+h_{34}, 2\left(h_{p}+q\right) ; z\right) .
$$

Here, $\chi_{q}\left(c, \boldsymbol{h}, h_{p}\right)$ is a well-definite sum over the level $q$ quasi-primaries and it involves the $1 \times 2 \rightarrow q, 3 \times 4 \rightarrow q$ fusion coefficients. It is completely fixed by Virasoro symmetry. In all cases $\chi_{0}=1$ and $\chi_{1}=0$. For the vacuum block, obtained with $h_{p} \rightarrow 0$, we need $h_{12}=h_{34}=0$. Setting now $\boldsymbol{h}=\left\{h_{1}, h_{3}\right\},(2.4)$ specialises to

$$
\mathcal{F}_{\mathrm{vac}}(c, \boldsymbol{h}, z)=\sum_{\substack{q=0 \\ q \text { even }}}^{\infty} \chi_{\mathrm{vac}, q}(c, \boldsymbol{h}) z^{q}{ }_{2} F_{1}(q, q, 2 q ; z)
$$

The first terms are rather simple

$$
\chi_{\mathrm{vac}, 2}(c, \boldsymbol{h})=\frac{2 h_{1} h_{3}}{c}, \quad \chi_{\mathrm{vac}, 4}(c, \boldsymbol{h})=\frac{10\left(h_{1}^{2}+\frac{h_{1}}{5}\right),\left(h_{3}^{2}+\frac{h_{3}}{5}\right)}{c(5 c+22)},
$$

and, for larger $q$, they are always rational functions of $c, h_{1}, h_{3}$ with rational numerical coefficients. Using the method of [15] to solve the $h_{p} \rightarrow 0$ limit of the recursion relation

$$
\begin{aligned}
\mathcal{F}\left(c, \boldsymbol{h}, h_{p}, z\right)= & z^{h_{p}}{ }_{2} F_{1}\left(h_{p}+h_{12}, h_{p}+h_{34}, 2 h_{p} ; z\right) \\
& +\sum_{m \geq 1, n \geq 2}^{\infty} \frac{R_{m n}\left(\boldsymbol{h}, h_{p}\right)}{c-c_{m n}\left(h_{p}\right)} \mathcal{F}\left(c_{m n}\left(h_{p}\right), \boldsymbol{h}, h_{p}+m n, z\right),
\end{aligned}
$$


we obtained the explicit rational functions $\chi_{\mathrm{vac}, q}(c, \boldsymbol{h})$ up to $q=16 .^{2}$ Notice that from the elementary relation

$$
{ }_{2} F_{1}\left(a, c-b, c ; \frac{z}{z-1}\right)=(1-z)^{a}{ }_{2} F_{1}(a, b, c ; z),
$$

we check invariance of (2.5) under the tranformation $z \rightarrow \frac{z}{z-1}$,

$$
\mathcal{F}_{\text {vac }}(c, \boldsymbol{h}, z)=\mathcal{F}_{\text {vac }}\left(c, \boldsymbol{h}, \frac{z}{z-1}\right),
$$

that is nothing but the (bootstrap) symmetry between the $s$ and $t$ scattering channels.

\section{Semiclassical limit}

As discussed in the introduction, the semiclassical limit of the vacuum block is defined as

$$
c \rightarrow \infty, \quad \lambda_{1}=\frac{h_{1}}{c}, \quad \lambda_{3}=\frac{h_{3}}{c} \text { fixed } .
$$

In this limit, there are arguments $[5,9,13,25]$ to expect that the block exponentiates according to the relation $\left(\boldsymbol{\lambda}=\left\{\lambda_{1}, \lambda_{3}\right\}\right)$

$$
\log \mathcal{F}(c, \boldsymbol{h}, z)=-\frac{c}{6} f(\boldsymbol{\lambda}, z)+\mathcal{O}\left(c^{0}\right) .
$$

The function $f(\boldsymbol{\lambda}, z)$ is not known in closed form, but it may be considered order by order at small values of one of the ratios $\lambda$. We define the coefficient functions $f^{(\ell)}(\lambda, z)$ by expanding in powers of $\lambda_{1}$

$$
f(\boldsymbol{\lambda}, z)=\sum_{\ell_{1}=1}^{\infty} \lambda_{1}^{\ell_{1}} f^{\left(\ell_{1}\right)}\left(\lambda_{3}, z\right) .
$$

The exact expression of the first function $f^{(1)}(\lambda, z)$ appearing in (3.3) is known from the calculation in [17] and reads

$$
\begin{aligned}
f^{(1)}(\lambda, z) & =12 \log \left(\frac{1-(1-z)^{\alpha}}{\alpha z}\right)+6(1-\alpha) \log (1-z), \\
\alpha & =\sqrt{1-24 \lambda} .
\end{aligned}
$$

In the following, it will be important to further refine the expansion (3.3) by separating out different powers of the ratio $\lambda_{3}$, as follows

$$
f^{\left(\ell_{1}\right)}\left(\lambda_{3}, z\right)=\sum_{\ell_{3}=1}^{\infty} \lambda_{3}^{\ell_{3}} f^{\left(\ell_{1}, \ell_{3}\right)}(z)
$$

\footnotetext{
${ }^{2}$ The expressions are available on request. Notice that they require some tricky implementation because a naive coding of the relations in [15] has a complexity that grows prohibitively on symbolic manipulation softwares. We warn the reader that the internal sum and product in (2.28) of [15] must be swapped.
} 
where we have the obvious symmetry $f^{\left(\ell, \ell^{\prime}\right)}(z)=f^{\left(\ell^{\prime}, \ell\right)}(z)$. In particular, from the result (3.4), we may obtain all the functions $f^{(1, \ell)}(z)$. The first cases are

$$
\begin{aligned}
f^{(1,1)}(z) & =-12 U_{2}, \\
f^{(1,2)}(z) & =12 U_{2}^{2}+\frac{72}{5} U_{3}, \\
f^{(1,3)}(z) & =-16 U_{2}^{3}-\frac{168}{5} U_{2} U_{3}-\frac{648}{35} U_{4}, \\
\ldots & \\
\text { with } \quad U_{q}(z) & =z^{q} \log ^{q-2}(1-z){ }_{2} F_{1}(q, q, 2 q ; z) .
\end{aligned}
$$

The function $f^{(2)}(\lambda, z)$ in (3.3) is not known and is the next-to-leading correction when one of the ratios $\boldsymbol{\lambda}$ is small. The main result of this paper is a closed form for this contribution, analogous to (3.4).

\subsection{Exact expansions from the recursion relations}

Using our explicit data for $\chi_{\mathrm{vac}, q}(c, \boldsymbol{h})$, we checked that the exponentiation property written in (3.2) holds perturbatively in small $z$ at least up to the order $\mathcal{O}\left(z^{17}\right)$. To clarify what one gets, we write here the first terms of the function $f(\boldsymbol{\lambda}, z){ }^{3}$

$$
\begin{aligned}
f(\boldsymbol{\lambda}, z)= & -12\left(\lambda_{1} \lambda_{3}\right) z^{2}-12\left(\lambda_{1} \lambda_{3}\right) z^{3}+\left(\frac{264}{5} \lambda_{3}^{2} \lambda_{1}^{2}-\frac{12}{5} \lambda_{3} \lambda_{1}^{2}-\frac{12}{5} \lambda_{3}^{2} \lambda_{1}-\frac{54 \lambda_{3} \lambda_{1}}{5}\right) z^{4} \\
& +\left(\frac{528}{5} \lambda_{3}^{2} \lambda_{1}^{2}-\frac{24}{5} \lambda_{3} \lambda_{1}^{2}-\frac{24}{5} \lambda_{3}^{2} \lambda_{1}-\frac{48 \lambda_{3} \lambda_{1}}{5}\right) z^{5} \\
& +\left(-\frac{24064}{35} \lambda_{3}^{3} \lambda_{1}^{3}+\frac{1776}{35} \lambda_{3}^{2} \lambda_{1}^{3}-\frac{32}{35} \lambda_{3} \lambda_{1}^{3}+\frac{1776}{35} \lambda_{3}^{3} \lambda_{1}^{2}+\frac{1014}{7} \lambda_{3}^{2} \lambda_{1}^{2}-\frac{234}{35} \lambda_{3} \lambda_{1}^{2}\right. \\
& \left.-\frac{32}{35} \lambda_{3}^{3} \lambda_{1}-\frac{234}{35} \lambda_{3}^{2} \lambda_{1}-\frac{60 \lambda_{3} \lambda_{1}}{7}\right) z^{6}+\left(-\frac{72192}{35} \lambda_{3}^{3} \lambda_{1}^{3}+\frac{5328}{35} \lambda_{3}^{2} \lambda_{1}^{3}-\frac{96}{35} \lambda_{3} \lambda_{1}^{3}\right. \\
& \left.+\frac{5328}{35} \lambda_{3}^{3} \lambda_{1}^{2}+\frac{1194}{7} \lambda_{3}^{2} \lambda_{1}^{2}-\frac{282}{35} \lambda_{3} \lambda_{1}^{2}-\frac{96}{35} \lambda_{3}^{3} \lambda_{1}-\frac{282}{35} \lambda_{3}^{2} \lambda_{1}-\frac{54 \lambda_{3} \lambda_{1}}{7}\right) z^{7}+\cdots
\end{aligned}
$$

We can collect the various powers of $\boldsymbol{\lambda}$ and extract series expansions for the functions $f^{\left(\ell, \ell^{\prime}\right)}(z)$ defined in (3.5). The first instances of the first order contributions $f^{(1, \ell)}(z)$ are

$$
\begin{aligned}
& f^{(1,1)}(z)=-12 z^{2}-12 z^{3}-\frac{54 z^{4}}{5}-\frac{48 z^{5}}{5}-\frac{60 z^{6}}{7}-\frac{54 z^{7}}{7}-7 z^{8}-\frac{32 z^{9}}{5}-\frac{324 z^{10}}{55}+\ldots, \\
& f^{(1,2)}(z)=-\frac{12 z^{4}}{5}-\frac{24 z^{5}}{5}-\frac{234 z^{6}}{35}-\frac{282 z^{7}}{35}-\frac{1578 z^{8}}{175}-\frac{1692 z^{9}}{175}-\frac{3886 z^{10}}{385}-\frac{3986 z^{11}}{385}+\ldots, \\
& f^{(1,3)}(z)=-\frac{32 z^{6}}{35}-\frac{96 z^{7}}{35}-\frac{908 z^{8}}{175}-\frac{1392 z^{9}}{175}-\frac{20826 z^{10}}{1925}-\frac{150 z^{11}}{11}-\frac{14293984 z^{12}}{875875}+\ldots
\end{aligned}
$$

\footnotetext{
${ }^{3}$ All other terms are again available on request, although they can be computed directly by evaluating the limit (3.1).
} 
One can check immediately that the expansions in (3.8) agree with the formulas in (3.6), as they should. Going further, from (3.7) — and including additional terms - we can write down the first cases of the second order contributions $f^{(2, \ell)}(z)$. They are

$$
\begin{aligned}
f^{(2,2)}(z)= & \frac{264 z^{4}}{5}+\frac{528 z^{5}}{5}+\frac{1014 z^{6}}{7}+\frac{1194 z^{7}}{7}+\frac{162516 z^{8}}{875}+\frac{169164 z^{9}}{875}+\frac{376744 z^{10}}{1925} \\
& +\frac{374564 z^{11}}{1925}+\frac{1171852056 z^{12}}{6131125}+\frac{1141630738 z^{13}}{6131125}+\frac{57446497 z^{14}}{318500} \\
& +\frac{121933377 z^{15}}{700700}+\frac{4487292704 z^{16}}{26801775}+\frac{439701599 z^{17}}{2734875}+\ldots
\end{aligned}
$$

and

$$
\begin{aligned}
f^{(2,3)}(z)= & \frac{1776 z^{6}}{35}+\frac{5328 z^{7}}{35}+\frac{250356 z^{8}}{875}+\frac{379824 z^{9}}{875}+\frac{801346 z^{10}}{1375}+\frac{1393606 z^{11}}{1925} \\
& +\frac{26149378198 z^{12}}{30655625}+\frac{29672482498 z^{13}}{30655625}+\frac{65496704213 z^{14}}{61311250}+\frac{2022337201 z^{15}}{1751750} \\
& +\frac{3838174474811 z^{16}}{3126873750}+\frac{2014459667366 z^{17}}{1563436875}+\ldots
\end{aligned}
$$

We have written many terms in (3.9) and (3.10) to emphasise that it is non trivial to identify their resummation in terms of a closed function of $z$ as it was possible in (3.6). The solution will be presented in the next section and indeed will be much more involved than the leading order (3.6). Here, we just notice that the relevant terms from (3.7) are

$$
\begin{aligned}
f^{(2)}(\lambda, z) & \left(\frac{264 \lambda^{2}}{5}-\frac{12 \lambda}{5}\right) z^{4}+\left(\frac{528 \lambda^{2}}{5}-\frac{24 \lambda}{5}\right) z^{5}+\left(\frac{1776 \lambda^{3}}{35}+\frac{1014 \lambda^{2}}{7}-\frac{234 \lambda}{35}\right) z^{6} \\
& +\left(\frac{5328 \lambda^{3}}{35}+\frac{1194 \lambda^{2}}{7}-\frac{282 \lambda}{35}\right) z^{7}+\left(\frac{36144 \lambda^{4}}{875}+\frac{250356 \lambda^{3}}{875}+\frac{162516 \lambda^{2}}{875}-\frac{1578 \lambda}{175}\right) z^{8} \\
& +\left(\frac{144576 \lambda^{4}}{875}+\frac{379824 \lambda^{3}}{875}+\frac{169164 \lambda^{2}}{875}-\frac{1692 \lambda}{175}\right) z^{9} \\
& +\left(\frac{301248 \lambda^{5}}{9625}+\frac{541336 \lambda^{4}}{1375}+\frac{801346 \lambda^{3}}{1375}+\frac{376744 \lambda^{2}}{1925}-\frac{3886 \lambda}{385}\right) z^{10} \\
& +\left(\frac{301248 \lambda^{5}}{1925}+\frac{1403848 \lambda^{4}}{1925}+\frac{1393606 \lambda^{3}}{1925}+\frac{374564 \lambda^{2}}{1925}-\frac{3986 \lambda}{385}\right) z^{11}+\ldots
\end{aligned}
$$

These may be used as a check, although we shall also provide much longer expansions at specialised values of $\lambda$ to further check. Of course, the terms linear in $\lambda$ in (3.11) define the function $f^{(2,1)}(z)$. This is equal to $f^{(1,2)}(z)$ and indeed the expansion is the same as that in the second line of (3.8). In the following, it will be convenient to trade $\lambda$ for the same $\alpha$ parameters as in (3.4), i.e. $\alpha=\sqrt{1-24 \lambda}$. With a little abuse of notation, we shall denote $f^{(2)}(\lambda(\alpha), z) \rightarrow f^{(2)}(\alpha, z)$ since no confusion should arise. The first terms of (3.11) 
after this redefinition are then

$$
\begin{aligned}
f^{(2)}(\alpha, z)= & \frac{1}{120}\left(\alpha^{2}-1\right)\left(11 \alpha^{2}+1\right) z^{4}+\frac{1}{60}\left(\alpha^{2}-1\right)\left(11 \alpha^{2}+1\right) z^{5} \\
& -\frac{\left(\alpha^{2}-1\right)\left(37 \alpha^{4}-2609 \alpha^{2}-236\right)}{10080} z^{6}-\frac{\left(\alpha^{2}-1\right)\left(37 \alpha^{4}-1069 \alpha^{2}-96\right)}{3360} z^{7} \\
& +\frac{\left(\alpha^{2}-1\right)\left(251 \alpha^{6}-42479 \alpha^{4}+734269 \alpha^{2}+65399\right)}{2016000} z^{8}+\ldots .
\end{aligned}
$$

Many additional terms are collected in appendix A.

\section{The exact expression for the NLO contribution $f^{(2)}(\lambda, z)$}

Our main result is the following closed expression for the derivative $\partial_{z} f^{(2)}(\lambda, z)$. This is what is obtained from the solution of the second order monodromy problem according to the procedure discussed in appendix B.

$$
\partial_{z} f^{(2)}(\lambda, z)=(\mathrm{I})+(\mathrm{II})+(\mathrm{III})
$$

with

$$
\begin{aligned}
& (\mathrm{I})=\frac{36}{(1-z) z\left(1-(1-z)^{\alpha}\right)^{3}}[(\alpha+1) z \\
& -(1-z)^{\alpha}(\alpha z+4 \alpha z \log (1-z)-8 \alpha z \log z-9 z+6) \\
& +(1-z)^{2 \alpha}(-\alpha z-4 \alpha z \log (1-z)+8 \alpha z \log z+3 z+8) \\
& \left.+(1-z)^{3 \alpha}((\alpha+3) z-2)\right] \text {, } \\
& (\mathrm{II})=\frac{144 \alpha\left((1-z)^{\alpha}+1\right)(1-z)^{\alpha-1}\left(\pi \cot (\pi \alpha)+2 \psi^{(0)}(\alpha)+2 \gamma\right)}{\left(1-(1-z)^{\alpha}\right)^{3}} \\
& (\mathrm{III})=-\frac{72\left((1-z)^{\alpha}+1\right)^{2}}{(1-z)\left(1-(1-z)^{\alpha}\right)^{3}}{ }_{2} F_{1}(1,-\alpha, 1-\alpha ; 1-z) \\
& -\frac{72\left((1-z)^{\alpha}+1\right)}{(\alpha-1) z\left(1-(1-z)^{\alpha}\right)^{2}}{ }_{2} F_{1}(1,-\alpha, 2-\alpha ; 1-z) \\
& +\frac{\left(-144(\alpha-1)(1-z)\left((z-1)^{2}\right)^{\alpha}+144(1-z)^{2 \alpha}\left(\alpha+\alpha z(1-z)^{\alpha}+z-1\right)\right)}{(\alpha+1) z\left(1-(1-z)^{\alpha}\right)^{3}} \times \\
& { }_{2} F_{1}(1, \alpha+1, \alpha+2 ; 1-z) \text {. }
\end{aligned}
$$

If we expand this expression in powers of $z$ and integrate term by term - with no additional integration constant — we recover indeed (3.12), including many additional non-trivial terms that may be found in appendix A.

\subsection{Exact expression for $f^{(2,2)}(z)$ and $f^{(2,3)}(z)$}

In order to obtain all the functions $f^{(2, \ell)}(z)$ from (4.1), we simply have to replace $\alpha=$ $\sqrt{1-24 \lambda}$ and expand around $\lambda=0$. The only non-trivial piece is (III) in (4.4). This may 
be treated by using the algorithms described in [33, 34]. We need the expansion of the hypergeometric functions in (4.4) around $\alpha=1$. Setting $\alpha=1+\varepsilon$, these read

$$
\begin{aligned}
{ }_{2} F_{1}(1,-\alpha, 1-\alpha, z)= & \frac{z}{\varepsilon}+z+z \log (1-z)+1+z\left(\log (1-z)-\operatorname{Li}_{2}(z)\right) \varepsilon \\
& -z\left(\operatorname{Li}_{2}(z)+\operatorname{Li}_{3}(z)\right) \varepsilon^{2}-z\left(\operatorname{Li}_{3}(z)+\operatorname{Li}_{4}(z)\right) \varepsilon^{3}+\ldots, \\
{ }_{2} F_{1}(1,-\alpha, 2-\alpha, z)= & 1-z+(-z-z \log (1-z)+\log (1-z)) \varepsilon \\
& -(z-1)\left(\log (1-z)-\operatorname{Li}_{2}(z)\right) \varepsilon^{2}+(z-1)\left(\operatorname{Li}_{2}(z)+\operatorname{Li}_{3}(z)\right) \varepsilon^{3}+\ldots, \\
{ }_{2} F_{1}(1,1+\alpha, 2+\alpha, z)= & -\frac{2(z+\log (1-z))}{z^{2}}+\frac{\left(-2 \operatorname{Li}_{2}(z)+z-\log (1-z)\right)}{z^{2}} \varepsilon \\
& -\frac{\left(\operatorname{Li}_{2}(z)-2 \operatorname{Li}_{3}(z)+z\right)}{z^{2}} \varepsilon^{2}+\frac{\left(\operatorname{Li}_{3}(z)-2 \operatorname{Li}_{4}(z)+z\right)}{z^{2}} \varepsilon^{3}+\ldots
\end{aligned}
$$

Using these results, we obtain

$$
\begin{aligned}
f^{(2,2)}(z)= & \frac{864}{z^{3}}\left[30 z^{3}+\log (1-z)(4(z-1) \log (1-z)(3 z-6 z \log z\right. \\
& \left.\left.+(4 z-6) \log (1-z))-3(z-2) z^{2}\right)\right] \\
& -\frac{41472(z-1)\left(-\operatorname{Li}_{3}(1-z)+\operatorname{Li}_{2}(1-z) \log (1-z)+\zeta_{3}\right)}{z^{2}} .
\end{aligned}
$$

Expansion in powers of $z$ reproduces the result in (3.9). Besides, the expression in (4.6) is crossing invariant, see (2.9). With more work, one can also derive the expression of $f^{(2,3)}(z)$, the main difficulty being the final integration of the expansion of (4.1). The result is

$$
\begin{aligned}
f^{(2,3)}(z)= & \frac{10368}{z^{4}}\left[2\left(23+12 \pi^{2}\right) z^{4}-3 z^{3} \log (1-z)(z+48 z \log (z)-2)\right. \\
& -12(z-1) z^{2} \log ^{2}(1-z)(2 \log (z)-1)+(z-1)(11(z-4) z+36) \log ^{4}(1-z) \\
& \left.-2(z-1) z \log ^{3}(1-z)(-9 z+12(z-2) \log (z)+14)\right] \\
& -\frac{497664}{z^{3}}\left[3 z^{3} \operatorname{Li}_{2}(z)+\operatorname{Li}_{2}(1-z)\left(3 z^{3}+(z-1) \log (1-z)(z+(z-2) \log (1-z))\right)\right. \\
& \left.-(z-1)(z+(z-2) \log (1-z))\left(\operatorname{Li}_{3}(1-z)-\zeta_{3}\right)\right] .
\end{aligned}
$$

Again, expansion in powers of $z$ reproduces the result in (3.10) and one checks that (4.7) is crossing invariant, see (2.9).

\section{A simple application: two interval Rényi entropy}

As a simple application, we follow the discussion in [15] and connect our results for the vacuum block with the two-interval Rényi entropy. This is the quantity $S_{n}$ obtained as the 4-point correlation functions of twist fields $\Phi_{ \pm}$

$$
S_{n}(z)=\frac{1}{1-n} \log \left\langle\Phi_{+}(\infty) \Phi_{-}(1) \Phi_{+}(z) \Phi_{-}(0)\right\rangle_{\mathscr{C}^{n} / \mathbb{Z}_{n}}, \quad h_{\Phi_{ \pm}}=\frac{n c}{24}\left(1-\frac{1}{n^{2}}\right),
$$


where $\mathscr{C}^{n} / \mathbb{Z}_{n}$ is a cyclic orbifold with central charge $n c$. At large $c$ the twist fields behave as heavy operators. Using the exponentiation (3.2) - taking into account the antiholomorphic part - the vacuum contribution to $S_{n}$ may be shown to be simply [27]

$$
S_{n, \operatorname{vac}}(z)=-\frac{n c}{3(1-n)} f\left(\frac{h_{\Phi}}{c}, \frac{h_{\Phi}}{c}, z\right)
$$

up to subleading corrections as $c \rightarrow \infty$. Replacing (3.7) into (5.2) we obtain the function $S_{n, \text { vac }}(z)$ at order $\mathcal{O}\left(z^{17}\right)$. The first 14 terms are shown is in appendix D. Of course, we reproduce the $\mathcal{O}\left(z^{9}\right)$ expansion in (3.15) of [35], with generic $n .{ }^{4}$

According to the analysis of [15], it is convenient to organise $S_{n}$ at fixed $z$ in an expansion around $\delta n=0$, where $n=1+\delta n$. Then, the expansions (3.3) and (3.5) resum an infinite number of terms. To this aim, we define the functions $S^{(k)}(z)$ by writing

$$
\left.S_{n, \operatorname{vac}}(z)\right|_{\mathcal{O}(c)}=\sum_{k=1}^{\infty} S^{(k)}(z)(\delta n)^{k} .
$$

The small $z$ expansion of the first five functions reads

$$
\begin{aligned}
& S^{(1)}(z)=-\frac{z^{2}}{36}-\frac{z^{3}}{36}-\frac{z^{4}}{40}-\frac{z^{5}}{45}-\frac{5 z^{6}}{252}-\frac{z^{7}}{56}-\frac{7 z^{8}}{432}+\ldots \\
& S^{(2)}(z)=\frac{z^{2}}{18}+\frac{z^{3}}{18}+\frac{53 z^{4}}{1080}+\frac{23 z^{5}}{540}+\frac{187 z^{6}}{5040}+\frac{493 z^{7}}{15120}+\frac{81 z^{8}}{2800}+\ldots \\
& S^{(3)}(z)=-\frac{13 z^{2}}{144}-\frac{13 z^{3}}{144}-\frac{25 z^{4}}{324}-\frac{83 z^{5}}{1296}-\frac{8267 z^{6}}{155520}-\frac{769 z^{7}}{17280}-\frac{8009 z^{8}}{212625}+\ldots \\
& S^{(4)}(z)=\frac{19 z^{2}}{144}+\frac{19 z^{3}}{144}+\frac{277 z^{4}}{2592}+\frac{53 z^{5}}{648}+\frac{9581 z^{6}}{155520}+\frac{2401 z^{7}}{51840}+\frac{316961 z^{8}}{9072000}+\ldots, \\
& S^{(5)}(z)=-\frac{13 z^{2}}{72}-\frac{13 z^{3}}{72}-\frac{871 z^{4}}{6480}-\frac{143 z^{5}}{1620}-\frac{147523 z^{6}}{2799360}-\frac{25843 z^{7}}{933120}-\frac{535277 z^{8}}{48988800}+\ldots
\end{aligned}
$$

The first four cases may be given in closed form using using our new results because

$$
\begin{aligned}
& S^{(1)}(z)=\frac{1}{432} f^{(1,1)}, \quad S^{(2)}(z)=\frac{1}{2592}\left(f^{(1,2)}-12 f^{(1,1)}\right), \\
& S^{(3)}(z)=\frac{1}{62208}\left(468 f^{(1,1)}-84 f^{(1,2)}+2 f^{(1,3)}+f^{(2,2)}\right), \\
& S^{(4)}(z)=\frac{1}{373248}\left(-4104 f^{(1,1)}+1188 f^{(1,2)}-60 f^{(1,3)}+f^{(1,4)}-30 f^{(2,2)}+f^{(2,3)}\right),
\end{aligned}
$$

and all relevant $f^{\left(\ell, \ell^{\prime}\right)}(z)$ have been computed. In particular, the terms $S^{(3)}$ and $S^{(4)}$ are an extension of the previous results since they involve $f^{(2,2)}$ and $f^{(2,3)}$. For completeness,

\footnotetext{
${ }^{4}$ The quantity $I_{n}$ in [35] is the mutual information equal to $-S_{n}$ plus a $\sim \log z$ term that is absent here due to our normalization, as discussed before.
} 
we give the explicit expressions of the combinations in (5.5)

$$
\begin{aligned}
& S^{(1)}=-\frac{(z-2) \log (1-z)-2 z}{6 z} \\
& S^{(2)}=\frac{\log (1-z)((z-2) z+2(z-1) \log (1-z))}{6 z^{2}}, \\
& S^{(3)}=\frac{1}{18 z^{3}}\left(12(z-1) z \operatorname{Li}_{3}(1-z)-12(z-1) z \operatorname{Li}_{2}(1-z) \log (1-z)+z^{3}-3 z^{3} \log (1-z)\right. \\
& -12 z^{2} \zeta_{3}+6 z^{2} \log ^{3}(1-z)-12 z^{2} \log ^{2}(1-z)-6 z^{2} \log ^{2}(1-z) \log (z)+6 z^{2} \log (1-z) \\
& \left.+12 z \zeta_{3}-16 z \log ^{3}(1-z)+10 \log ^{3}(1-z)+12 z \log ^{2}(1-z)+6 z \log ^{2}(1-z) \log (z)\right), \\
& S^{(4)}=-\frac{1}{18 z^{4}}\left(72 z^{4} \operatorname{Li}_{2}(z)+36 z^{3} \operatorname{Li}_{3}(1-z)-24 z^{3} \operatorname{Li}_{3}(1-z) \log (1-z)-36 z^{2} \operatorname{Li}_{3}(1-z)\right. \\
& +72 z^{2} \operatorname{Li}_{3}(1-z) \log (1-z)+12 z \operatorname{Li}_{2}(1-z)\left(6 z^{3}+2\left(z^{2}-3 z+2\right) \log ^{2}(1-z)\right. \\
& -3(z-1) z \log (1-z))-48 z \operatorname{Li}_{3}(1-z) \log (1-z)-12 \pi^{2} z^{4}-3 z^{4} \log (1-z) \\
& +72 z^{4} \log (1-z) \log (z)-36 z^{3} \zeta_{3}+24 z^{3} \zeta_{3} \log (1-z)-6 z^{3} \log ^{4}(1-z) \\
& +18 z^{3} \log ^{3}(1-z)+12 z^{3} \log ^{3}(1-z) \log (z)-18 z^{3} \log ^{2}(1-z)-18 z^{3} \log ^{2}(1-z) \log (z) \\
& +6 z^{3} \log (1-z)+36 z^{2} \zeta_{3}-72 z^{2} \zeta_{3} \log (1-z)+31 z^{2} \log ^{4}(1-z)-48 z^{2} \log ^{3}(1-z) \\
& -36 z^{2} \log ^{3}(1-z) \log (z)+18 z^{2} \log ^{2}(1-z)+18 z^{2} \log ^{2}(1-z) \log (z)+48 z \zeta_{3} \log (1-z) \\
& \left.-46 z \log ^{4}(1-z)+21 \log ^{4}(1-z)+30 z \log ^{3}(1-z)+24 z \log ^{3}(1-z) \log (z)\right) \text {. }
\end{aligned}
$$

Of course, their expansions at small $z$ agree with the first four lines in (5.4). We conclude this section with a curious remark. The definition (5.1) of the Rényi entropy requires $n$ to be integer. Analytic continuation is possible at least at the level of the perturbative expansion in $z$, since all coefficients are rational functions of $n$, see appendix D. At the special value $n=1 / 2$, we found that it is possible to resum our long expansion giving the closed form

$$
\begin{aligned}
\left.S_{\frac{1}{2}, \operatorname{vac}}(z)\right|_{\mathcal{O}(c)}= & \frac{1}{6} \log \left(\frac{16}{27(1-z)}\right)-\frac{1}{3} \sinh ^{-1}\left(\frac{2 z-1}{\sqrt{3}}\right) \\
& +\frac{2}{3} \tanh ^{-1}\left(\frac{2 z-1}{4 \sqrt{1-z(1-z)}+3}\right)
\end{aligned}
$$

\section{Conclusions}

In this paper we have improved our knowledge of the semiclassical heavy-light limit by computing the next-to-leading order corrections to the vacuum Virasoro block. Generally speaking, these corrections have an interesting dual interpretation at the level of the background geometry. Our explicit results may be discouraging due to their complexity, but could be tested in some special limit. A natural extension of this work is to CFTs with extended $\mathcal{W}$-symmetry, see [36]. This is a non-trivial task because of the dependence on the additional $\mathcal{W}$-charges besides Virasoro conformal weights. Another generalization is to consider blocks with a non-trivial intermediate field with $h_{p} \neq 0$. This may be possible by exploiting the recent results in [37] about the evaluation of Wilson lines in 3D higher spin 
gravity. The idea is that if the chiral Wilson line carries a spin- $s$ charge, we can extract its part linear in this charge. This term must be proportional to the Virasoro block for the spin- $s$ current exchange. Finally, we believe that it could be of some interest to provide a cleaner derivation of the perturbative solution of the monodromy equations, possibly exploiting its relation with the gravity equations of motion in Chern-Simons form.

\section{Acknowledgments}

We thank E. Perlmutter for useful comments.

\section{A Long expansion for the function $f^{(2)}(\alpha, z)$}

Let us write the expansion (3.12) in the form

$$
f^{(2)}(\alpha, z)=\sum_{n=4}^{\infty}\left(\alpha^{2}-1\right) p_{n}(\alpha) z^{n} .
$$

Then, we have the following explicit polynomials $p_{n}(\alpha)$

$$
\begin{aligned}
p_{4}= & \frac{1}{120}\left(11 \alpha^{2}+1\right) \\
p_{5}= & \frac{1}{60}\left(11 \alpha^{2}+1\right) \\
p_{6}= & \frac{-37 \alpha^{4}+2609 \alpha^{2}+236}{10080}, \\
p_{7}= & \frac{-37 \alpha^{4}+1069 \alpha^{2}+96}{3360}, \\
p_{8}= & \frac{251 \alpha^{6}-42479 \alpha^{4}+734269 \alpha^{2}+65399}{2016000} \\
p_{9}= & \frac{251 \alpha^{6}-16579 \alpha^{4}+201569 \alpha^{2}+17799}{504000} \\
p_{10}= & \frac{-1569 \alpha^{8}+479945 \alpha^{6}-18258687 \alpha^{4}+170711655 \alpha^{2}+14943856}{399168000} \\
p_{11}= & \frac{-1569 \alpha^{8}+181757 \alpha^{6}-4716675 \alpha^{4}+35862963 \alpha^{2}+3112564}{79833600} \\
p_{12}= & \frac{1}{15256200960000}\left(1815004 \alpha^{10}-873963991 \alpha^{8}+56886151422 \alpha^{6}-1106810435488 \alpha^{4}\right. \\
& \left.+7108850079574 \alpha^{2}+611825905479\right) \\
p_{13}= & \frac{1}{2542700160000}\left(1815004 \alpha^{10}-324264841 \alpha^{8}+14101619632 \alpha^{6}-218412314878 \alpha^{4}\right. \\
& \left.+1217781886564 \alpha^{2}+103957984919\right) \\
p_{14}= & \frac{1}{122049607680000}\left(-427265 \alpha^{12}+297527391 \alpha^{10}-29352540329 \alpha^{8}+940279163283 \alpha^{6}\right. \\
& \left.-12079216519482 \alpha^{4}+59671526161226 \alpha^{2}+5053911319976\right) \\
p_{15}= & \frac{1}{3487131648000}\left(-85453 \alpha^{12}+21753395 \alpha^{10}-1412547837 \alpha^{8}+35474720295 \alpha^{6}\right. \\
& \left.-389381682962 \alpha^{4}+1731385519410 \alpha^{2}+145526223952\right) \\
&
\end{aligned}
$$




$$
\begin{aligned}
p_{16}= & \frac{1}{2240830797004800000}\left(226205741 \alpha^{14}-215001129589 \alpha^{12}+29836328448647 \alpha^{10}\right. \\
& -140864185677413 \alpha^{8}+28952603091655483 \alpha^{6}-277672065926382407 \alpha^{4} \\
& \left.+1125312507357000129 \alpha^{2}+93890464607856909\right) \\
p_{17}= & \frac{1}{280103849625600000}\left(226205741 \alpha^{14}-77720885089 \alpha^{12}+7019243713307 \alpha^{10}\right. \\
& -256841114655053 \alpha^{8}+4457607960596863 \alpha^{6}-38008205857100927 \alpha^{4} \\
& \left.+141820189774444089 \alpha^{2}+11748922612161069\right) .
\end{aligned}
$$

\section{B Details of the derivation}

\section{B.1 The monodromy problem}

The results of section 4 are obtained by the monodromy method [13, 17, 27]. Let us briefly recall the main points. The function $f(\boldsymbol{\lambda}, z)$ is related to the monodromy properties of the following equation specialised to the vacuum block case

$$
\psi^{\prime \prime}(w)+T(\boldsymbol{\lambda}, z ; w) \psi(w)=0,
$$

where

$$
\begin{aligned}
T(\boldsymbol{\lambda}, z ; w)= & \frac{6 \lambda_{3}}{(1-w)^{2}}+6 \lambda_{1}\left(\frac{1}{w^{2}}+\frac{1}{(w-z)^{2}}+\frac{2}{w(1-w)}\right) \\
& -c(\boldsymbol{\lambda}, z) \frac{z(1-z)}{w(w-z)(1-w)} .
\end{aligned}
$$

The accessory parameter $c(\boldsymbol{\lambda}, z)$ is fixed by imposing a trivial monodromy along a contour encircling the two points $w=0, z$. Then, the semiclassical Virasoro block $f(\boldsymbol{\lambda}, z)$ is obtained by integrating the relation ${ }^{5}$

$$
c(\boldsymbol{\lambda} ; z)-\frac{12 \lambda_{1}}{z}=\partial_{z} f(\boldsymbol{\lambda}, z)
$$

with suitable boundary condition at $z \rightarrow 0$. The determination of the accessory parameter is a difficult analytical problem. ${ }^{6}$ In our case, there is no known solution for generic $\boldsymbol{\lambda}$, but one can work out a perturbative expansion in powers of $\lambda_{1}$ at generic $\lambda_{3}$. Plugging (3.3) into (B.3), and assuming that $\psi(w)$ admits a regular expansion around $\lambda_{1}=0$, we set

$$
\begin{aligned}
\psi(w) & =\psi_{0}(w)+\lambda_{1} \psi_{1}(w)+\lambda_{1}^{2} \psi_{2}(w)+\ldots, \\
c(\boldsymbol{\lambda}, z) & =\lambda_{1} c_{1}\left(\lambda_{3}, z\right)+\lambda_{1}^{2} c_{2}\left(\lambda_{3}, z\right)+\ldots
\end{aligned}
$$

and solve the equations

$$
\begin{aligned}
& \psi_{0}^{\prime \prime}(w)+T_{0}\left(\lambda_{3} ; w\right) \psi_{0}=0, \\
& \psi_{1}^{\prime \prime}(w)+T_{0}\left(\lambda_{3} ; w\right) \psi_{1}=-T_{1}\left(\lambda_{3}, z ; w\right) \psi_{0}, \\
& \psi_{2}^{\prime \prime}(w)+T_{0}\left(\lambda_{3} ; w\right) \psi_{2}=-T_{2}\left(\lambda_{3}, z ; w\right) \psi_{0}-T_{1}\left(\lambda_{3}, z ; w\right) \psi_{1},
\end{aligned}
$$

\footnotetext{
${ }^{5}$ The second term in the l.h.s. of (B.3) is due to a normalization factor $z^{2 h_{1}}$ that we must take into account when matching the monodromy computation with our definition of the Virasoro block.

${ }^{6}$ Rigorous results that are somewhat related to our problem are presented in [38-40] with applications in [41].
} 
where

$$
\begin{aligned}
T_{0}\left(\lambda_{3} ; w\right) & =\frac{6 \lambda_{3}}{(1-w)^{2}}, \\
T_{1}\left(\lambda_{3}, z ; w\right) & =6\left(\frac{1}{w^{2}}+\frac{1}{(w-z)^{2}}+\frac{2}{w(1-w)}\right)-c_{1}\left(\lambda_{3}, z\right) \frac{z(1-z)}{w(w-z)(1-w)}, \\
T_{2}\left(\lambda_{3}, z ; w\right) & =-c_{2}\left(\lambda_{3}, z\right) \frac{z(1-z)}{w(w-z)(1-w)} .
\end{aligned}
$$

The solution at first order is well known and quite simple. One starts from the two independent solutions at leading order, i.e. (B.5a). They are

$$
\psi_{0}^{ \pm}(w)=(1-w)^{\frac{1}{2}(1 \pm \alpha)}, \quad \alpha=\sqrt{1-24 \lambda_{3}} .
$$

Two linearly independent solutions to (B.5b) are then

$$
\psi_{1}^{ \pm}(w)=\frac{1}{\alpha} \psi_{0}^{+} \int d w \psi_{0}^{-} T_{1} \psi_{0}^{ \pm}-\frac{1}{\alpha} \psi_{0}^{-} \int d w \psi_{0}^{+} T_{1} \psi_{0}^{ \pm} .
$$

The integrands in (B.8) have only polar singularities around $w=0$ and $w=z$. Thus, $\psi_{1}^{ \pm}$have trivial monodromy around these two points when the sum of residues in $w=$ $0, z$ vanishes. This gives immediately (we identify again with little abuse of notation $\left.c_{n}\left(\lambda_{3}(\alpha), z\right) \rightarrow c_{n}(\alpha, z)\right)$

$$
c_{1}(\alpha, z)=6 \frac{\alpha-1+(1-z)^{\alpha}(1+\alpha)}{(1-z)\left(1-(1-z)^{\alpha}\right)} .
$$

Integrating (B.9) with the condition $f^{(1)}\left(\lambda_{3}, z\right)=\mathcal{O}\left(z^{2}\right)$ for $z \rightarrow 0$, one gets (3.4). In principle, one could solve (B.5c) in the very same way, but $\psi_{1}^{ \pm}$will be integrated and it is rather difficult to control the analytic structure of the result. Indeed, some of the integrals in (B.8) are definitely non trivial, like for example

$$
\begin{aligned}
\int d w\left[\psi_{0}^{+}(w)\right]^{2} & \left(\frac{1}{w^{2}}+\frac{1}{(w-z)^{2}}+\frac{2}{w(1-w)}\right)= \\
= & -(1-w)^{\alpha+1}\left(\frac{{ }_{2} F_{1}\left(1,1,1-\alpha ; \frac{1}{w}\right)}{\alpha w}-\alpha \Gamma(\alpha+1)_{2} \tilde{F}_{1}(1, \alpha+1, \alpha+2 ; 1-w)\right. \\
& \left.+\frac{(w-1) \Gamma(-\alpha)_{2} \tilde{F}_{1}\left(1,2,1-\alpha ; \frac{z-1}{z-w}\right)}{(w-z)^{2}}+\frac{1}{w}\right) .
\end{aligned}
$$

In the next subsection, we discuss what can be obtained at specialised values of the parameter $\alpha$, see (B.7), where the function $c_{2}(\alpha, z)$ is first expanded in powers of $z$ and then resummed, thus bypassing the above problems.

\section{B.2 Special cases}

If we fix $\alpha=\sqrt{1-24 \lambda_{3}}$ and work out the solution of (B.5c) order by order around $z=0$, we see that $\psi_{1}^{ \pm}$in (B.8) have only poles. The procedure leading to (B.9) can then be 
repeated and it is possible to derive very long series expansions of the form

$$
c_{2}(\alpha, z)=\sum_{n=0}^{\infty} c_{2, n} z^{n}
$$

We analysed these expansions for the special values

$$
\alpha=\frac{1}{2}, \frac{1}{3}, \frac{1}{4}, \frac{1}{5}, \frac{1}{6}, \frac{1}{7}
$$

and found that in all cases, the function $C(\alpha, t)=c_{2}\left(\alpha, 1-(1-t)^{\frac{1}{\alpha}}\right)$ is the generating function of a holonomic sequence, i.e. it obeys a differential equation of the form [42]

$$
P(\alpha, t) \partial_{t} C(\alpha, t)+Q(\alpha, t) C(\alpha, t)+R(\alpha, t)=0,
$$

where $P, Q, R$ are polynomials in $t$. Once these polynomials are identified, the function $c_{2}(\alpha, z)$ may be determined by integrating (B.13) and replacing $t=1-(1-z)^{\alpha}$. We identified the precise form of (B.13) for the special values in (B.12) by computing the coefficients $c_{2, n}$ in (B.11) up to $n \sim 60$. Just to give an example, for $\alpha=\frac{1}{2}$, one finds the differential equation

$$
t(t-1)^{3}(t-2)^{2} \partial_{t} C\left(\frac{1}{2}, t\right)+(t-1)^{2}(t-2)\left(3 t^{2}-10 t+6\right) C\left(\frac{1}{2}, t\right)-18 t^{3}=0,
$$

leading to

$$
\begin{aligned}
c_{2}\left(\frac{1}{2}, z\right)= & -\frac{18(z-6 \sqrt{1-z}-2)}{(z-1) z} \\
& -\frac{72((\sqrt{1-z}+2) z-2(\sqrt{1-z}+1))(4 \log (\sqrt{1-z}+1)-\log (16(1-z)))}{(z-1) z(z+2 \sqrt{1-z}-2)} .
\end{aligned}
$$

As a check, one can expand (B.15) in powers of $z$ and integrate term by term recovering the expansion (3.12) for $\alpha=\frac{1}{2}$. Of course, after some manipulation, one can also check a posteriori that (B.15) is in agreement with (4.1). Unfortunately, inspection of $c_{2}(\alpha, z)$ for the values (B.12) reveals that it is far from being trivial to identify a regularity. For instance, for $\alpha=\frac{1}{3}$, one finds by the same procedure the function

$$
\begin{aligned}
c_{2}\left(\frac{1}{3}, z\right)= & \frac{24\left(-z+5(1-z)^{2 / 3}+5 \sqrt[3]{1-z}+2\right)}{(z-1) z} \\
& -\frac{144(\sqrt[3]{1-z}+1)\left(-z+(1-z)^{2 / 3}+\sqrt[3]{1-z}+1\right) \log \left(\frac{1}{3}\left(\sqrt[3]{1-z}+\frac{1}{\sqrt[3]{1-z}}+1\right)\right)}{(\sqrt[3]{1-z}-1)^{2}(z-1) z},
\end{aligned}
$$

and one may hope to guess a general formula. This works easily for the first lines of (B.15) and (B.16), i.e. for the non-transcendental part of the result. Instead, the logarithmic parts 
are not that simple, despite their compact form. For instance, for $\alpha=\frac{1}{5}$ one has

$$
\begin{aligned}
c_{2}\left(\frac{1}{5}, z\right)= & -\frac{72(2 \sqrt[5]{1-z} z+7 z+5 \sqrt[5]{1-z}-5)}{5(\sqrt[5]{1-z}-1)(z-1) z} \\
& +\frac{72(\sqrt[5]{1-z}+1)}{5(\sqrt[5]{1-z}-1)^{3}(1-z)^{4 / 5}}\left[-5 \log \left((1-z)^{4 / 5}+(1-z)^{3 / 5}+(1-z)^{2 / 5}+\sqrt[5]{1-z}+1\right)\right. \\
& +2 \log (1-z)+5 \log 5]+\frac{144(\sqrt[5]{1-z}+1) \operatorname{coth}^{-1}\left(\frac{\sqrt{5}\left((1-z)^{2 / 5}+1\right)}{(\sqrt[5]{1-z}-1)^{2}}\right)}{\sqrt{5}(\sqrt[5]{1-z}-1)^{3}(1-z)^{4 / 5}}
\end{aligned}
$$

and the appearance of the inverse hyperbolic cotangent becomes a major problem in the identification of some regularity. The explicit results for $\alpha=\frac{1}{4}, \frac{1}{6}$ are collected for the reader's interest in appendix $\mathrm{C}$ in simplified form in order to display their structure. Of course, they may be also recovered from (4.1).

Further explicit results may be obtained by the very same procedure for integer values

$$
\alpha=2,3,4, \ldots
$$

Here, we do not care about the physical interpretation of these values and simply use them to explore the form of $c_{2}(\alpha, z)$ at more specific points. In all cases in (B.18), $c_{2}(\alpha, z)$ is simply a rational function. Explicit expressions are

$$
\begin{aligned}
c_{2}(2, z)= & \frac{36 z^{3}}{(z-2)^{3}(z-1)} \\
c_{2}(3, z)= & \frac{72(z-2) z^{3}\left(z^{2}-5 z+5\right)}{(z-1)\left(z^{2}-3 z+3\right)^{3}} \\
c_{2}(4, z)= & \frac{12 z^{3}\left(9 z^{6}-98 z^{5}+466 z^{4}-1208 z^{3}+1784 z^{2}-1416 z+472\right)}{(z-2)^{3}(z-1)\left(z^{2}-2 z+2\right)^{3}} \\
c_{2}(5, z)= & \frac{12(z-2) z^{3}}{(z-1)\left(z^{4}-5 z^{3}+10 z^{2}-10 z+5\right)^{3}}\left(12 z^{8}-147 z^{7}+826 z^{6}-2783 z^{5}\right. \\
& \left.+6104 z^{4}-8875 z^{3}+8325 z^{2}-4600 z+1150\right)
\end{aligned}
$$

and so on. Despite their relative simplicity, it is again non trivial to find a regularity in the sequence (B.19a)-(B.19d).

\section{B.3 Back to the monodromy problem}

We now come back to the second order monodromy problem in (B.5c) and try to exploit the many exact results of section B.2 in order to set up a mixed heuristic strategy. Two independent solutions may be written as in (B.8) and read

$$
\psi_{2}^{ \pm}(w)=\frac{1}{\alpha} \psi_{0}^{+} \int d w \psi_{0}^{-}\left(T_{1} \psi_{1}^{ \pm}+T_{2} \psi_{0}^{ \pm}\right)-\frac{1}{\alpha} \psi_{0}^{-} \int d w \psi_{0}^{+}\left(T_{1} \psi_{1}^{ \pm}+T_{2} \psi_{0}^{ \pm}\right) .
$$

Expanding around $w=0$ or $w=z$, one finds

$$
\begin{aligned}
& \psi_{2}^{ \pm}(w)=\frac{1}{2} \log ^{2} w+A^{ \pm}(\alpha, z) \log w+\mathcal{O}(w \log w), \\
& \psi_{2}^{ \pm}(w)=B^{ \pm}(\alpha, z) \log ^{2}(w-z)+C^{ \pm}(\alpha, z) \log (w-z)+\mathcal{O}((w-z) \log (w-z)) .
\end{aligned}
$$


The monodromy cannot be read from these local expansions. Nevertheless, one observes that the integrand in the second term of (B.20) for $\psi_{2}^{+}$has a remarkable property. Its expansion around $w=0$ has the form

$$
\psi_{0}^{+}\left(T_{1} \psi_{1}^{+}+T_{2} \psi_{0}^{+}\right)=\frac{-\log w+f_{1}(\alpha, z)}{w^{2}}+\frac{f_{2}\left(\alpha, z, c_{2}\right)}{w}+\ldots,
$$

with no $\log w$ in the residue of the simple pole. Also, the function $f_{2}\left(\alpha, z, c_{2}\right)$ involves $c_{2}(\alpha, z)$ that is absent from the double pole. A similar property holds for the expansion around $w=z$

$$
\psi_{0}^{+}\left(T_{1} \psi_{1}^{+}+T_{2} \psi_{0}^{+}\right)=\frac{-(1-z)^{\alpha+1} \log (w-z)+\widetilde{f}_{1}(\alpha, z)}{(w-z)^{2}}+\frac{\widetilde{f}_{2}\left(\alpha, z, c_{2}\right)}{w-z}+\ldots,
$$

Then, we determine $c_{2}(\alpha, z)$ by the simple and well posed requirement that the sum of the simple pole residues in (B.22) and (B.23) vanishes, let us call it $\widehat{c}_{2}$

$$
f_{2}\left(\alpha, z, \widehat{c}_{2}\right)+\widetilde{f}_{2}\left(\alpha, z, \widehat{c}_{2}\right)=0
$$

Admittedly, this procedure has no particular rigorous motivation but just tries to get the most out of the expansions (B.22) and (B.23). In particular, there is no reason why $\widehat{c}_{2}$ has to be identified with the exact accessory function $c_{2}$ such that the exact monodromy is trivial. However, one can now compare this function $\widehat{c}_{2}$ with the exact solutions determined in section B.2 at the many special values (B.12), (B.18). Quite surprisingly, the mismatch is always very simple

$$
c_{2}(\alpha, z)=\widehat{c}_{2}(\alpha, z)+18(\alpha+1) \frac{1+(1-z)^{\alpha}}{(1-z)\left[1-(1-z)^{\alpha}\right]} .
$$

In other words, the term $\widehat{c}_{2}(\alpha, z)$ captures all the transcendental contributions whose origin is from hypergeometric functions depending on $\alpha$. The remainder is the very simple function in the second term of (B.25). Its origin is presumably from the contributions of the logarithms in the double pole of (B.22) and (B.23), although a global analysis of the exact $\psi_{2}^{ \pm}$would be necessary to clarify this. The combination in the r.h.s. of (B.25) is precisely the result in (4.1). It passes various non-trivial checks:

1. It reproduces the exact result at the specialized points (B.12) and (B.18).

2. It agrees with the expansion (3.12) at order $\mathcal{O}\left(z^{16}\right)$ for generic $\alpha$. We recall that (3.12) comes from the higher order solution of the Zamolodchikov recursion relations.

3. Finally, it is non perturbatively crossing symmetric, see (2.9).

Item (2) in the above list is perhaps the most stringent one because $\alpha$ is generic and the expansion (3.12) - including all terms in appendix A - depends on it in a highly nontrivial way. All these features lead us to conclude that (4.1) is the correct expression for the next-to-leading semiclassical vacuum block. In principle, it may be possible to make our derivation rigorous, but we feel that little doubts are left about the validity of (4.1). 


\section{Expressions of $c_{2}(\alpha, z)$ at $\alpha=\frac{1}{4}, \frac{1}{6}$}

We report here, for the reader's interest, the specialized function $c_{2}(\alpha, z)$ for $\alpha=\frac{1}{4}, \frac{1}{6}$. They can be obtained from (4.1), but we show them to display their structure.

$$
\begin{aligned}
c_{2}\left(\frac{1}{4}, z\right)= & \frac{9\left(2\left(7(1-z)^{3 / 4}+7 \sqrt{1-z}+7 \sqrt[4]{1-z}+3\right)-3 z\right)}{(z-1) z} \\
& -\frac{36}{(\sqrt[4]{1-z}-1)^{2}(z-1) z}[(2(\sqrt[4]{1-z}+1)(\sqrt{1-z}+1)-(\sqrt[4]{1-z}+2) z) \\
& (4 \log (\sqrt[4]{1-z}+1)+2 \log (\sqrt{1-z}+1)-\log (64(1-z)))] \\
c_{2}\left(\frac{1}{6}, z\right)= & -\frac{6(12(\sqrt[6]{1-z}-1)+(5 \sqrt[6]{1-z}+17) z)}{(\sqrt[6]{1-z}-1)(z-1) z} \\
& -\frac{24}{(\sqrt[6]{1-z}-1)^{3}(1-z)^{5 / 6}}[(\sqrt[6]{1-z}+1)(4 \log (\sqrt[6]{1-z}+1) \\
& +\log (\sqrt[3]{1-z}-\sqrt[6]{1-z}+1) \\
& +3 \log (\sqrt[3]{1-z}+\sqrt[6]{1-z}+1)-\log (432(1-z)))]
\end{aligned}
$$

\section{Long expansion of the two-interval Rényi entropy}

Using (5.2) and our extended data for the expansion (3.7), we get

$$
S_{n, \mathrm{vac}}=\frac{c(n-1)(n+1)^{2}}{n^{3}} \sum_{k=2}^{\infty} \sigma_{k} z^{k}+\mathcal{O}\left(c^{0}\right),
$$

with the explicit coefficients

$$
\begin{aligned}
\sigma_{2} & =-\frac{1}{144}, \\
\sigma_{3} & =-\frac{1}{144}, \\
\sigma_{4} & =\frac{-1309 n^{4}+2 n^{2}+11}{207360 n^{4}}, \\
\sigma_{5} & =\frac{-589 n^{4}+2 n^{2}+11}{103680 n^{4}}, \\
\sigma_{6} & =\frac{-805139 n^{8}+4244 n^{6}+23397 n^{4}+86 n^{2}-188}{156764160 n^{8}}, \\
\sigma_{7} & =\frac{-244439 n^{8}+1724 n^{6}+9537 n^{4}+86 n^{2}-188}{52254720 n^{8}}, \\
\sigma_{8} & =\frac{-6459666587 n^{12}+56285106 n^{10}+312586347 n^{8}+4722748 n^{6}-10301973 n^{4}-67854 n^{2}+58213}{1504935936000 n^{12}} \\
\sigma_{9} & =\frac{-1491872987 n^{12}+15293106 n^{10}+85282347 n^{8}+1833148 n^{6}-3985173 n^{4}-67854 n^{2}+58213}{376233984000 n^{12}}, \\
\sigma_{10} & =\frac{1}{297977315328000 n^{16}}\left(-1098074352431 n^{16}+12818273224 n^{14}+71779112743 n^{12}+2005530358 n^{10}\right. \\
& \left.-4341928013 n^{8}-129198788 n^{6}+110407921 n^{4}+844006 n^{2}-445820\right), \\
\sigma_{11} & =\frac{1}{59595463065600 n^{16}}\left(-205168198115 n^{16}+2665356736 n^{14}+14986883227 n^{12}+514319494 n^{10}\right. \\
& \left.-1108414169 n^{8}-48588236 n^{6}+41250877 n^{4}+844006 n^{2}-445820\right),
\end{aligned}
$$




$$
\begin{aligned}
\sigma_{12} & =\frac{1}{1366643159020339200000 n^{20}}\left(-4415649574925892347 n^{20}+62766476909113526 n^{18}\right. \\
& +354351390797880981 n^{16}+14372653961026536 n^{14}-30825885554247939 n^{12}-1808908135564014 n^{10} \\
& \left.+1523003046143151 n^{8}+56147131454316 n^{6}-29483342794674 n^{4}-232322730364 n^{2}+88390810828\right), \\
\sigma_{13} & =\frac{1}{227773859836723200000 n^{20}}\left(-693492488278292747 n^{20}+10647680903036726 n^{18}\right. \\
& +60348261746649381 n^{16}+2814211318880136 n^{14}-6006073680991539 n^{12}-443967283088814 n^{10} \\
& \left.+370233764078751 n^{8}+20663431202316 n^{6}-10740178354674 n^{4}-232322730364 n^{2}+88390810828\right), \\
\sigma_{14} & =\frac{1}{16399717908244070400000 n^{24}}\left(-47220015602598891185 n^{24}+775231178323314996 n^{22}\right. \\
& +4410547598118951299 n^{20}+231637262931595986 n^{18}-491900587819492959 n^{16}-43930432559597964 n^{14} \\
& +36253571634577911 n^{12}+2776290861193866 n^{10}-1423709558722344 n^{8}-56824093196764 n^{6} \\
& \left.+21453757559406 n^{4}+166480749880 n^{2}-49440922128\right), \\
\sigma_{15} & =\frac{1}{2342816844034867200000 n^{24}}\left(-6399731741802376253 n^{24}+111442004278820940 n^{22}\right. \\
& +636367936312062263 n^{20}+37045104581062770 n^{18}-78277417467172875 n^{16}-8194459617128580 n^{14} \\
& +6688019137439955 n^{12}+659887041494970 n^{10}-333028465921200 n^{8}-20581747259980 n^{6} \\
& \left.+7664791070238 n^{4}+166480749880 n^{2}-49440922128\right),
\end{aligned}
$$

and so on.

Open Access. This article is distributed under the terms of the Creative Commons Attribution License (CC-BY 4.0), which permits any use, distribution and reproduction in any medium, provided the original author(s) and source are credited.

\section{References}

[1] S. Ferrara, A.F. Grillo and R. Gatto, Manifestly conformal-covariant expansion on the light cone, Phys. Rev. D 5 (1972) 3102 [inSPIRE].

[2] S. Ferrara, A.F. Grillo and R. Gatto, Manifestly conformal covariant operator-product expansion, Lett. Nuovo Cim. 2S2 (1971) 1363 [INSPIRE].

[3] S. Ferrara, A.F. Grillo, G. Parisi and R. Gatto, Covariant expansion of the conformal four-point function, Nucl. Phys. B 49 (1972) 77 [INSPIRE].

[4] S. Ferrara, R. Gatto and A.F. Grillo, Properties of Partial Wave Amplitudes in Conformal Invariant Field Theories, Nuovo Cim. A 26 (1975) 226 [INSPIRE].

[5] A.A. Belavin, A.M. Polyakov and A.B. Zamolodchikov, Infinite Conformal Symmetry in Two-Dimensional Quantum Field Theory, Nucl. Phys. B 241 (1984) 333 [inSPIRE].

[6] F.A. Dolan and H. Osborn, Conformal four point functions and the operator product expansion, Nucl. Phys. B 599 (2001) 459 [hep-th/0011040] [INSPIRE].

[7] F.A. Dolan and H. Osborn, Conformal partial waves and the operator product expansion, Nucl. Phys. B 678 (2004) 491 [hep-th/0309180] [INSPIRE].

[8] R. Rattazzi, V.S. Rychkov, E. Tonni and A. Vichi, Bounding scalar operator dimensions in 4D CFT, JHEP 12 (2008) 031 [arXiv:0807.0004] [INSPIRE].

[9] D. Harlow, J. Maltz and E. Witten, Analytic Continuation of Liouville Theory, JHEP 12 (2011) 071 [arXiv:1108.4417] [INSPIRE]. 
[10] L.F. Alday, D. Gaiotto and Y. Tachikawa, Liouville Correlation Functions from Four-dimensional Gauge Theories, Lett. Math. Phys. 91 (2010) 167 [arXiv:0906.3219] [INSPIRE].

[11] V.A. Alba, V.A. Fateev, A.V. Litvinov and G.M. Tarnopolskiy, On combinatorial expansion of the conformal blocks arising from AGT conjecture, Lett. Math. Phys. 98 (2011) 33 [arXiv: 1012.1312] [INSPIRE].

[12] P.D. Francesco, P. Mathieu and D. Sénéchal, Conformal field theory, Graduate texts in contemporary physics, Springer (1999).

[13] A.B. Zamolodchikov, Conformal symmetry in two-dimensions: an explicit recurrence formula for the conformal partial wave amplitude, Commun. Math. Phys. 96 (1984) 419 [INSPIRE].

[14] A.B. Zamolodchikov and A.B. Zamolodchikov, Structure constants and conformal bootstrap in Liouville field theory, Nucl. Phys. B 477 (1996) 577 [hep-th/9506136] [INSPIRE].

[15] E. Perlmutter, Virasoro conformal blocks in closed form, JHEP 08 (2015) 088 [arXiv: 1502.07742] [INSPIRE].

[16] A. Litvinov, S. Lukyanov, N. Nekrasov and A. Zamolodchikov, Classical Conformal Blocks and Painleve VI, JHEP 07 (2014) 144 [arXiv:1309.4700] [INSPIRE].

[17] A.L. Fitzpatrick, J. Kaplan and M.T. Walters, Universality of Long-Distance AdS Physics from the CFT Bootstrap, JHEP 08 (2014) 145 [arXiv:1403.6829] [INSPIRE].

[18] M. Bañados, C. Teitelboim and J. Zanelli, The black hole in three-dimensional space-time, Phys. Rev. Lett. 69 (1992) 1849 [hep-th/9204099] [INSPIRE].

[19] E. Hijano, P. Kraus and R. Snively, Worldline approach to semi-classical conformal blocks, JHEP 07 (2015) 131 [arXiv: 1501.02260] [INSPIRE].

[20] A.L. Fitzpatrick, J. Kaplan and M.T. Walters, Virasoro Conformal Blocks and Thermality from Classical Background Fields, JHEP 11 (2015) 200 [arXiv: 1501.05315] [INSPIRE].

[21] A.L. Fitzpatrick, J. Kaplan, M.T. Walters and J. Wang, Eikonalization of Conformal Blocks, JHEP 09 (2015) 019 [arXiv: 1504.01737] [INSPIRE].

[22] A.L. Fitzpatrick, J. Kaplan, M.T. Walters and J. Wang, Hawking from Catalan, arXiv: 1510.00014 [INSPIRE].

[23] E. Hijano, P. Kraus, E. Perlmutter and R. Snively, Semiclassical Virasoro blocks from AdS $S_{3}$ gravity, JHEP 12 (2015) 077 [arXiv: 1508.04987] [INSPIRE].

[24] E. Hijano, P. Kraus, E. Perlmutter and R. Snively, Witten Diagrams Revisited: The AdS Geometry of Conformal Blocks, JHEP 01 (2016) 146 [arXiv:1508.00501] [INSPIRE].

[25] Al.B. Zamolodchikov, Two-dimensional conformal symmetry and critical four-spin correlation functions in the ashkin-teller model, Sov. Phys. JETP 63 (1986) 1061.

[26] A.L. Fitzpatrick and J. Kaplan, Conformal Blocks Beyond the Semi-Classical Limit, arXiv: 1512.03052 [INSPIRE].

[27] T. Hartman, Entanglement Entropy at Large Central Charge, arXiv:1303.6955 [INSPIRE].

[28] K.B. Alkalaev and V.A. Belavin, Conformal blocks of $W_{N}$ minimal models and AGT correspondence, JHEP 07 (2014) 024 [arXiv: 1404.7094] [INSPIRE].

[29] K.B. Alkalaev and V.A. Belavin, Classical conformal blocks via AdS/CFT correspondence, JHEP 08 (2015) 049 [arXiv: 1504.05943] [INSPIRE]. 
[30] K.B. Alkalaev and V.A. Belavin, Monodromic vs geodesic computation of Virasoro classical conformal blocks, arXiv:1510.06685 [INSPIRE].

[31] T. Faulkner, The Entanglement Renyi Entropies of Disjoint Intervals in AdS/CFT, arXiv:1303.7221 [INSPIRE].

[32] J.M. Figueroa-O'Farrill and S. Schrans, The Spin 6 Extended Conformal Algebra, Phys. Lett. B 245 (1990) 471 [INSPIRE].

[33] T. Huber and D. Maître, HypExp: A Mathematica package for expanding hypergeometric functions around integer-valued parameters, Comput. Phys. Commun. 175 (2006) 122 [hep-ph/0507094] [INSPIRE].

[34] T. Huber and D. Maître, HypExp 2, Expanding Hypergeometric Functions about Half-Integer Parameters, Comput. Phys. Commun. 178 (2008) 755 [arXiv:0708.2443] [INSPIRE].

[35] B. Chen, J. Long and J.-j. Zhang, Holographic Rényi entropy for CFT with $W$ symmetry, JHEP 04 (2014) 041 [arXiv: 1312.5510] [INSPIRE].

[36] J. de Boer, A. Castro, E. Hijano, J.I. Jottar and P. Kraus, Higher spin entanglement and $\mathcal{W}_{\mathrm{N}}$ conformal blocks, JHEP 07 (2015) 168 [arXiv:1412.7520] [INSPIRE].

[37] A. Hegde, P. Kraus and E. Perlmutter, General Results for Higher Spin Wilson Lines and Entanglement in Vasiliev Theory, JHEP 01 (2016) 176 [arXiv:1511.05555] [INSPIRE].

[38] P. Menotti, Accessory parameters for Liouville theory on the torus, JHEP 12 (2012) 001 [arXiv:1207.6884] [INSPIRE].

[39] P. Menotti, Hyperbolic deformation of the strip-equation and the accessory parameters for the torus, JHEP 09 (2013) 132 [arXiv:1307.0306] [INSPIRE].

[40] P. Menotti, On the monodromy problem for the four-punctured sphere, J. Phys. A 47 (2014) 415201 [arXiv: 1401.2409 ] [INSPIRE].

[41] M. Kulaxizi, A. Parnachev and G. Policastro, Conformal Blocks and Negativity at Large Central Charge, JHEP 09 (2014) 010 [arXiv: 1407.0324] [INSPIRE].

[42] D. Zeilberger, A holonomic systems approach to special functions identities, J. Comput. Appl. Math. 32 (1990) 321. 